\title{
CIRURGIA METABÓLICA: MUDANÇAS NA ANATOMIA GASTROINTESTINAL E A REMISSÃO DO DIABETES MELLITUS TIPO 2
}

\author{
Metabolic surgery: changes in gastrointestinal anatomy and type 2 diabetes mellitus remission
}

\author{
Ricardo COHEN, Marcelo Cerdan TORRES, Carlos A SCHIAVON
}

Trabalho realizado no Centro de Excelência no Tratamento Cirúrgico da Obesidade e Síndrome Metabólica do Hospital Alemão Oswaldo Cruz. São Paulo, SP, Brasil.

DESCRITORES - Cirurgia bariátrica. Obesidade mórbida. Diabetes mellitus. Derivação gástrica.

\section{Correspondência:}

Ricardo Cohen,

e-mail: rvcohen@mac.com

Fonte de financiamento: não há

Conflito de interesses: não há

Recebido para publicação: 11/11/2009

Aceito para publicação: 01/03/2010

HEADINGS - Bariatric surgery. Obesity, morbid. Diabetes mellitus. Gastric bypass.
RESUMO - Introdução - O diabetes mellitus tipo 2 (DMT2) é uma das maiores causas de morte no mundo devido a sua relação direta com as doenças cardiovasculares. Apesar dos avanços da terapêutica, a maioria dos pacientes nunca atinge os objetivos terapêuticos necessários para prevenção de suas complicações. A cirurgia metabólica abre um campo para novas perspectivas no controle desta doença. Objetivos - Descrever o tratamento cirúrgico de pacientes portadores de diabetes mellitus tipo 2, e os resultados encontrados após os procedimentos, além de discutir os mecanismos das alterações metabólicas encontradas no pós-operatório destes pacientes. Métodos - As bases de dados PubMed e Lilacs foram revisadas utilizandose os seguintes descritores: cirurgia bariátrica, obesidade móbida, diabetes mellitus e bypass gástrico. Resultados - Procedimentos convencionais sobre o trato gastrointestinal para o tratamento da obesidade mórbida demonstraram grande melhora do DMT2, sem relação direta com a perda ponderal. Estudos ilustraram que o re-arranjo da anatomia gastrointestinal é o mediador primário do controle cirúrgico do diabetes. Todas operações bariátricas melhoram o DMT2, mas essa melhora acontece por mecanismos fisiológicos distintos. Conclusão - Assim como as novas tendências na literatura sobre diabetes, deve-se buscar estudos randomizados e controlados, procedimento cirúrgico versus o melhor tratamento clínico, focando no controle glicêmico, de lipídeos e da pressão arterial. Esses estudos, além de demonstrar o papel potencial da cirurgia para diabetes, podem definir o melhor momento para a indicação cirúrgica.

ABSTRACT - Introduction - Type 2 diabetes mellitus ( T2DM), is the major cause of mortality worldwide, mainly secondary to cardiovascular events. In spite of the recent advances in the medical treatment, an expressive number of patients remain uncontrolled. Metabolic surgery may open new frontiers to control this devastating disease. Aim - To describe the surgical alternatives to treat surgically T2DM patients, including some proposed mechanisms of action that are behind the metabolic improvement. Methods - PubMed and Lilacs database were searched according to the following keywords: bariatric surgery, morbid obesity, diabetes mellitus, gastric bypass. Results - Some conventional procedures over the gastrointestinal tract have an important effect on T2DM control, without a direct effect of weight loss. Some studies report that rearranging the gastrointestinal tract anatomy is the key factor that lead to that beneficial metabolic effect of surgery. All bariatric operations lead to T2DM improvement, but with distinct mechanisms underlying that effect. Conclusion - There is a trend to stimulate research in this new exciting field. Randomized controlled trials are demanded, comparing surgical versus the best medical treatment of T2DM. After those results, the exact role of gastrointestinal tract surgery will be defined and its benefit over medical treatment as well.

\section{INTRODUÇÃO}

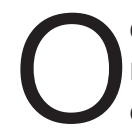
diabetes mellitus tipo 2 (DMT2) é uma das maiores causas de morte no mundo devido à sua relação direta com as doenças cardiovasculares, cerebrovasculares e insuficiência renal. Além disso, é responsável por um grande número de complicações como a cegueira, as amputações, a disfunção erétil, a diarréia e a gastroparesia8. O tratamento clínico para essa doença avançou consideravelmente, mas continua deixando a maioria dos pacientes susceptíveis às suas graves complicações. 
Enquanto novas drogas continuam a melhorar o tratamento clínico, a maioria dos pacientes nunca atinge os objetivos definidos para o sucesso da terapêutica, o que abre espaço a opção de novas possibilidades de tratamento como a cirurgia.

\section{O tratamento cirúrgico do DMT2: resultados após operações bariátricas}

A resolução clínica do DMT2, definida como a independência de todas medicações anti-diabéticas ${ }^{12}$, ocorreu em $48 \%$ dos pacientes submetidos à banda gástrica ajustável, $84 \%$ após bypass gástrico em $Y$ de Roux e $98 \%$ após derivação bílio-pancreática ${ }^{4}$. A resolução do DMT2 após banda gástrica é proporcional à perda de peso. A resolução que ocorre após os dois últimos procedimentos tipicamente ocorre muito rapidamente para ser atribuída apenas à perda de peso, sugerindo que pode existir um efeito direto e mais profundo sobre a homeostase da glicose. $O$ efeito anti-diabético da cirurgia bariátrica perdura por longos períodos, tendo em vista que grandes séries de bypass gástrico em $Y$ de Roux demonstraram controle glicêmico e níveis normais de hemoglobina glicada até com 14 anos de seguimento.

Enquanto o DMT2 é frequentemente associado à obesidade, esta relação é dependente da localização geográfica. Interessante notar que não existem estudos publicados que demonstrem alguma evidência que obesidade é causa de diabetes. O índice de massa corporal (IMC) médio de pacientes diabéticos no Estados Unidos é 31, enquanto na Índia é 27. Apesar da efetividade em relação à perda de peso e resolução de comorbidades, a cirurgia bariátrica é, em teoria, menos desejável para pacientes com peso normal ou sobrepeso. Cohen, et at.5 publicaram recentemente o tratamento cirúrgico de 37 pacientes fora dos padrões de indicação cirúrgica, definidos pelo NIH (Instituto Nacional de Saúde, EUA) em 1991, com IMC entre 32 e 35, todos com DMT2, além de outras comorbidade. Esses pacientes foram submetidos à bypass gástrico em $Y$ de Roux e todos apresentaram remissão do diabetes.

Então, se a resolução do DMT2 ocorre muito antes de uma perda de peso considerável e é duradoura, por que não oferecer o tratamento cirúrgico para paciente com IMC mais baixo?

Mecanismos prováveis para o controle glicêmico Independente do entendimento da explicação molecular, que ainda está por ser elucidada, será muito importante entender qual parte da nova anatomia originada do re-arranjo pós bypass gástrico em $Y$ de Roux ou derivação bílio-pancreática é essencial para o efeito sobre o diabetes.

\section{Mecanismos do intestino proximal e distal}

O mecanismo do intestino proximal afirma que a exclusão do duodeno e do jejuno proximal do trânsito alimentar poderia inibir a secreção de um possível sinal que promove a resistência insulínica, levando ao controle do DMT2.

Uma proposta alternativa, o mecanismo do intestino distal, justifica a remissão do DMT2 como resultante de um contato rápido do bolo alimentar com o intestino distal, aumentando um sinal fisiológico que melhora o metabolismo glicídico. O potencial candidato a mediador desse efeito é o GLP-1 e/ou peptídeos do intestino distal.

Apesar de não ser simples identificar moléculas responsáveis por esses efeito com o conhecimento atual, se essas teorias se mostrarem verdadeiras, podere-se abrir novas oportunidades na busca da causa e da cura do diabetes.

\section{Neoglicogênese intestinal}

Recentemente, um grupo francês publicou um trabalho muito elegante comparando o efeito de dois tipos de operações sobre o controle do DMT2: banda gástrica e exclusão duodenal. O grupo da exclusão duodenal especificamente reduziu a ingestão alimentar e aumentou a sensibilidade à insulina, medida pela produção endógena de glicose. A neoglicogênese intestinal aumentou após o procedimento de exclusão duodenal, mas não após a banda gástrica. Eles forneceram uma evidência mecanística que o rearranjo da anatomia do intestino proximal leva a um efeito benéfico na ingestão alimentar e na homeostase da glicose envolvendo a neoglicogênese intestinal, independente dos níveis de GLP1 ou alteração no peso. Eles aventaram a possibilidade de um importante caminho de sensores hepatoportais ${ }^{15}$.

\section{glicose \\ Alterações de mecanismos transportadores de}

Em setembro de $2009^{14}$, em trabalho experimental, foi demonstrado que a exclusão duodenal reduz significantemente a estrutura celular intestinal e o transporte de glicose, com diminuição da capacidade absortiva após a reconstrução em $Y$ de Roux. Esses achados abrem outra fronteira na explicação da ação antidiabética após a exclusão da passagem de alimentos pelo intestino proximal.

\section{Cura, controle ou remissão?}

O diabetes tipo 2 tem fisiopatologia muito complexa que inclui produção inadequada de glicose pelo fígado, genética, deposição pancreática de amilóide, resistência insulínica e falta de um efeito incretínico através de vários mecanismos entre outros. Como é muito difícil entender completamente todos mecanismos relacionados a como a cirurgia pode contribuir para melhorar o DMT2, o termo cura não deveria ser usado para descrever os resultados pósoperatórios.

A cirurgia, assim como outras formas de tratamento, deve ser interpretada como terapêutica complementar e, não como terapia exclusiva. Nunca 
dever-se-ia esquecer que algumas drogas e, até a insulina, são muito úteis na obtenção do controle do DMT2, principalmente na fase inicial do pós-operatório. Entre essas, metformina, pioglitazona e sulfoniluréias podem ser necessárias para manter níveis adequados de glicose.

Em declaração consensual da Sociedade Americana de Diabetes (ADA) definindo cura do DMT2 foi publicada em novembro de $2009^{1}$. Os autores concordaram com as seguintes definições, que são as mesmas para o diabetes tipo 1 e tipo 2:

- Remissão é definida como a obtenção de glicemia abaixo da faixa para diabetes na ausência de terapia farmacológica adequada (medicações anti-hiperglicêmicas ou imunossupressoras após transplante), grande esforço na mudança do estilo de vida, história de operação bariátrica/metabólica ou procedimentos em desenvolvimento (repetidas trocas de dispositivos endoluminais).

- Remissão pode ser caracterizada como parcial ou completa. A remissão parcial significa hiperglicemia sub-diabética (A1C não diagnóstica para diabetes [<6,5\%] e glicemia de jejum entre 100 e 125 mg/ $\mathrm{dL}[5,6-6,9 \mathrm{mmol} / \mathrm{L}]$ ) por pelo menos um ano de duração na ausência de terapia farmacológica ativa ou procedimentos em desenvolvimento. A remissão completa é o retorno às medidas normais do metabolismo da glicose (A1C na faixa normal, glicemia de jejum < 100mg/dL [5,6 mmol/L]) por pelo menos 1 ano de duração na ausência de terapia farmacológica ativa ou procedimentos em desenvolvimento.

- Glicemia não diabética resultante de medicações de uso contínuo ou repetidos procedimentos não se adequaria à definição de remissão, tendo em vista que essas intervenções são consideradas tratamento.

- Remissão pode ser considerada como resultado de um dispositivo implantável (banda gástrica, dispositivos endoluminais) apenas após o paciente ter alcançado a estabilidade, não necessitando mais de repetidos ajustes e/ou troca de dispositivos.

- Remissão prolongada é a que dura mais do que cinco anos e pode ser considerada operacionalmente com cura. O período de cinco anos foi escolhido arbitrariamente, tendo em vista que não existe dado atuarial indicando a probabilidade de recidiva durante vários períodos de tempo a partir do início da normoglicemia. É reconhecido que o risco de recidiva provavelmente se mantém mais alto para pessoas com diabetes do que para indivíduos pareados por idade, sexo, IMC e raça que nunca tiveram diabetes.

\section{Relatos recentes em humanos}

Bypass duodeno-jejunal (Figura 1)

Cohen, et al.6 publicaram no início de 2007 dois casos de pacientes com diabetes que se submeteram a bypass duodenojejunal, foi um passo na direção de estender o achados dos estudos animais para a arena clínica. Os pacientes apresentavam IMC de 29 e 30,3 kg/

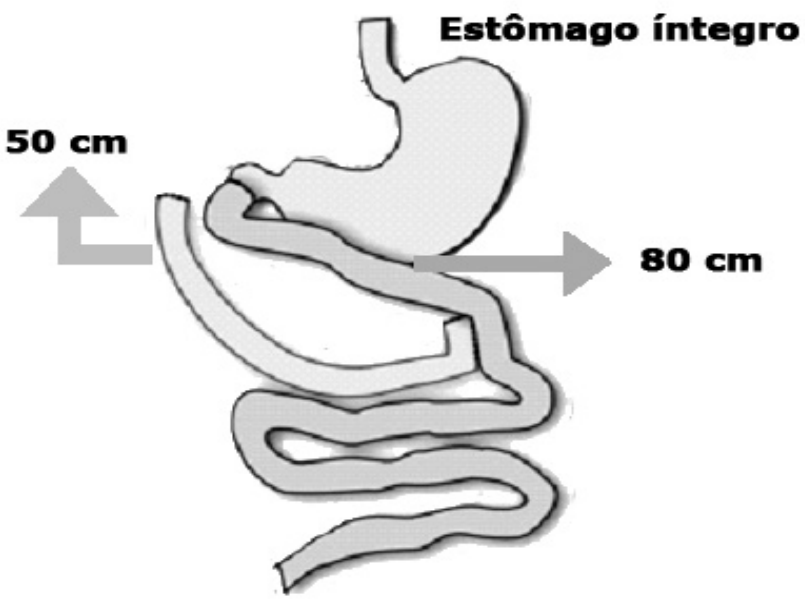

FIGURA 1 - Esquema de bypass duodenojejunal

$\mathrm{m}^{2}$. A história de diabetes não era muito longa (dois e sete anos, respectivamente), e era tratado com insulina mais metformina em um caso, e com rosiglitazona no outro. Apesar de os dados laboratoriais pré-operatórios não estarem apresentados, as avaliações em uma semana, um mês, e, depois disso, todos meses por nove meses demonstraram melhora rápida e inequívoca em várias medidas simples do controle glicêmico. As glicemias de jejum estavam inicialmente na faixa para diabetes (148 e $178 \mathrm{mg} / \mathrm{dL}$ ), mas elas decresceram rapidamente após a operação, alcançando níveis não diabéticos em um mês e se mantiveram em $100 \mathrm{mg} / \mathrm{dL}$ do terceiro ao nono mês de pós-operatório. Da mesma forma, a insulina de jejum no início era alta (27 e 29 $\mathrm{mmol} / \mathrm{L}$ ), mas declinaram rápida e progressivamente após a operação, permanecendo com níveis típicos de pessoas não diabéticas (aproximadamente $5 \mathrm{mmol} / \mathrm{L}$ ) do terceiro ao nono mês de pós-operatório. Refletindo a melhora da glicemia, a hemoglobina A1c foi de níveis diabéticos (8-9\%) para níveis normais (5-6\%) em três meses e permaneceram igualmente baixos nos seis meses seguintes de pós-operatório. Um paciente recebeu alta após alguns dias de pós-operatório sem medicações anti-diabéticas e, o outro, descontinuou suas medicações com cinco semanas de pós-operatório. Resumidamente, ambos pacientes passaram de diabetes mal controlado, apesar das medicações, para situação de normoglicemia sem medicações. Um achado importante foi que essa transformação ocorreu sem perda de peso nos dois pacientes.

Após esses bons resultados, os autores deste artigo iniciaram seu primeiro protocolo, no qual 86 pacientes foram submetidos à bypass duodenojejunal. O IMC desses pacientes variou entre 22 e 34. Para

\begin{tabular}{|l|}
\hline - Resolução - Sem medicações ou insulina, HbA1c $<7$ \\
\hline - Controle - Menos medicações, sem insulina, HbA1c $<7$ \\
\hline - Melhora - Menos medicações, sem insulina, HbA1c $<$ basal \\
\hline - Sem resposta - Igual ou pior que o basal \\
\hline
\end{tabular}

FIGURA 2 - Normalização para classificar os resultados 
avaliar os resultados, foram seguidos arbitrariamente os critérios descritos na Figura 2.

Naqueles com seguimento mais longo (12 meses), 78\% demonstraram melhora ou remissão do DMT2, independente de ganho ou perda de peso. Não há dúvida que esse achados são forte mudança de paradigma no tratamento do diabetes.

Nessa série, houve pacientes com resposta excelente, sem insulina ou medicações, que na verdade, até ganharam peso após a operação e continuam sem diabetes. Também não houve aumento significativo do GLP-1.

$\mathrm{Na}$ avaliação da qualidade de vida pelo SF 36, foram demonstrados altos índices de satisfação, $86 \%$ dos pacientes no sexto mês e $80 \%$ no 12 o. mês, principalmente relacionada às funções físicas, sociais e à vitalidade.

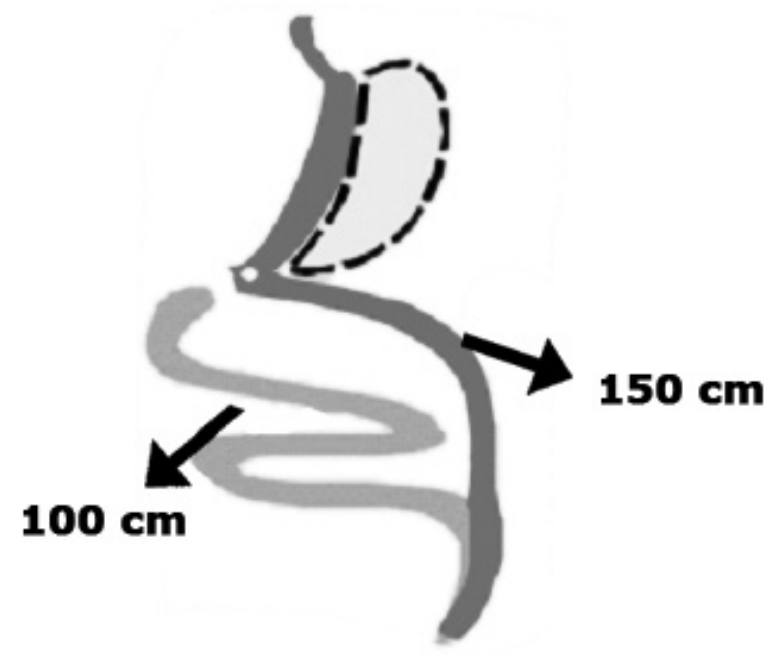

FIGURA 3 - Esquema do "switch" duodenal curto

Bypass duodeno-jejunal com gastrectomia em manga (Sleeve) (Figura 3)

Existem várias questões não respondidas no mecanismo de ação para controle do diabetes após o rearranjo anatômico do trato gastrointestinal superior, mesmo assim, baseado em estudos sobre a gastrectomia vertical, estes autores decidiram modificar a técnica, introduzindo a ressecção gástrica ao procedimento, consequentemente acrescentando outros mecanismos no controle do diabetes.

Esses estudos sugerem que a grelina é responsável por acelerar o esvaziamento gástrico, aumentando o aporte de glicose para o intestino ${ }^{2,3}$.

Alguns autores, principalmente de áreas da ciência básica, descreveram em diabéticos que a grelina tem a capacidade bioquímica de diminuir a produção pancreática de insulina. Dessa forma, a remoção da maior parte do local de produção da grelina - o fundo gástrico - consegue-se diminuir o esvaziamento gástrico, diminuindo o aporte de glicose no intestino, além de retirar um possível bloqueador da produção de insulina.

Ressecando apenas o fundo e parte do corpo gástrico e preservando o piloro também parece ser uma etapa chave na diminuição dos picos de glicemia após a ingestão alimentar, levando à melhor resposta de primeira fase da insulina e melhores resultados glicêmicos.

Alguns importantes estudos clínicos como Accord, Advance e o Va Diabetes demonstraram que pacientes diabéticos deveriam ser tratados com objetivo não focado apenas em um controle glicêmico rigoroso, mas também em todas sua comorbidades, como hipertensão arterial e dislipidemia ${ }^{7}$.

O Steno Trial, publicado em 2009, demonstrou de forma elegante que focando-se intensivamente na glicemia, na pressão arterial e no controle lipídico, pode-se proporcionar diminuição significativa na mortalidade da população diabética.

Baseado na literatura citada anteriormente, os autores deste trabalho decidiram conduzir um segundo protocolo e passaram a realizar o bypass duodenojejunal com gastrectomia em manga, o qual poder-se-ia chamar de "duodenal switch curto", adicionando uma gastrectomia em manga calibrada com uma sonda de $50 \mathrm{~F}$ em 47 pacientes.

Os objetivos primários do estudo foram controle da glicemia de jejum e pós-prandial e, os secundários, o controle de lipídeos e da hipertensão arterial, além da medida da espessura da camada íntima carotídea, como um importante marcador de arteriosclerose.

Adicionalmente, baseado em estudos dos autores de comprimento da alças e resolução do diabetes na população superobesa ${ }^{11}$, aumentou-se a alça bíliopancreática para $100 \mathrm{~cm}$ e a alimentar para $150 \mathrm{~cm}$.

Além destes achados, Patti ${ }^{10}$ apresentou em 2007 trabalho mostrando que após as modificações anatômicas do bypass gástrico em $Y$ de Roux existe alteração da circulação enterohepática de ácidos biliares. Os dados de Patti sugerem que os níveis alterados de ácidos biliares e a sua composição podem contribuir na melhora do metabolismo glicídico e lipídico em pacientes que se submeteram ao bypass gástrico com alças mais longas.

O acréscimo da gastrectomia diminui a incidência de náusea e vômitos para próximo de zero, enquanto no primeiro protocolo era de $20 \%$.

Com seguimento médio de um ano, encontrouse que a associação da gastrectomia e o aumento do comprimento das alças não acrescentou nenhuma perda ponderal a esse grupo de pacientes mais magros (perda total de peso de 6\%) e, além disso, conseguiuse resolução ou controle do DMT2 em $71 \%$ dos casos e em $100 \%$, se incluir-se os que obtiveram melhora.

Esse quadro é promissor, afinal, precocemente no pós-operatório, os pacientes apresentam melhora do diabetes como um efeito implícito e direto do procedimento realizado.

Como objetivos secundários, alcançou-se, em 12 meses, $67 \%$ de controle da hipertensão (níveis normais, sem medicação), normalização de triglicerídeos e LDL colesterol em $77 \%$ e $81 \%$ respectivamente. A espessura 
íntima reduziu significativamente após 12 meses quando comparada com os dados iniciais.

Ação antidiabética e perda ponderal: causa e efeito ou ação do rearranjo da anatomia gastrointestinal?

Em 2008, Laferrere, et al. ${ }^{9}$ compararam dois grupos de 10 pacientes, um pela perda de peso pósoperatória e outro pós-dieta, cada um combinado pelo IMC e história de DMT2,. Em pacientes com perda de peso semelhantes, eles compararam o efeito incretínico nos dois grupos. Concluíram que a liberação incretínica era seis vezes maior no grupo cirúrgico e o seu efeito não estava diretamente relacionado à perda de peso, mas sim, à perda de peso induzida pela operação no primeiro mês.

Os achados do estudo em animais e no de Laferrere são boas evidências de que o efeito incretínico e o potencial controle do metabolismo da glicose podem não estar diretamente relacionados à perda de peso isolada.

Será o IMC tão importante como se imaginava?

Não há duvida que os achados descritos acima são forte mudança no paradigma do tratamento dos pacientes com diabetes. Nessas séries, observou-se fortes respondedores, ou seja, pacientes sem insulina ou medicações que na verdade re-ganharam peso após a operação, mas continuavam livres do diabetes. De fato, o IMC sozinho não é ferramenta ideal para avaliar-se detalhadamente a relação risco-benefício dos pacientes com diabetes. Hoje, não existe evidência científica de que haja um valor definido de corte para o IMC que possa distinguir para quem a operação resolverá o diabetes e para quais pacientes ela será inefetiva.

\section{Recomendações propostas}

No fim de marco de 2007, um grupo de aproximadamente 53 profissionais envolvidos com DMT2, reuniram-se em Roma para uma conferência de consenso sobre os próximos passos que deveriam ser seguidos para alcançar o sucesso no campo da Cirurgia Metabólica. Entre os especialistas, havia cientistas de áreas básicas, endocrinologistas, diabetologistas e cirurgiões. O evento foi chamado 2007 Diabetes Surgery Summit. Várias apresentações ocorreram nos dois primeiros dias e, no terceiro, após um painel de votação eletrônica entre os especialistas, algumas definições sobre o futuro da Cirurgia Metabólica foram divulgadas. Para validar qualquer definição, isto é, para chamá-la de consenso, deveriam receber votação de pelo menos $70 \%$ dos especialistas envolvidos. As principais definições com consenso foram ${ }^{13}$ :

1. Todas as operações bariátricas melhoram o DMT2, entretanto, só algumas delas apresentam os critérios para defini-las como operações anti-diabéticas (100\%).

2. Modificações anatômicas de várias regiões do trato gastrointestinal parecem contribuir para a melhora do DMT2 através de distintos mecanismos fisiológicos (83\%).

3. O bypass gastrointestinal pode melhorar o diabetes por mecanismos que estão além da mudança na ingestão alimentar e do peso corporal (98\%).

4. A cirurgia gastrointestinal pode ser apropriada para o tratamento do DMT2 em pacientes que sejam candidatos apropriados à operação com IMC entre 30 e 35 e que estejam inadequadamente controlados pela terapia medicamentosa e mudanças de estilo de vida (82\%).

5. A colaboração entre endocrinologistas, cirurgiões e investigadores de ciências básicas deveria ser encorajada para facilitar o entendimento dos mecanismos pelos quais o trato gastrointestinal regula o metabolismo e para permitir o uso desses mecanismos para melhora do tratamento do DMT2 (100\%).

CONCLUSÕES

Procedimentos convencionais sobre 0 trato gastrointestinal para o tratamento da obesidade mórbida demonstraram melhora importante do DMT2, resultando em glicemia e níveis de $\mathrm{HbAlc}$ normais com a suspensão de todos medicamentos relacionados ao diabetes ou insulina. Muitas vezes, o retorno à euglicemia de jejum, níveis normais de glicemia pósprandial e níveis regulares da insulina foi observado em dias ou semanas após a operação, sugerindo que a perda de peso sozinha não consegue explicar inteiramente porque o procedimento cirúrgico melhora o diabetes. Apesar de que a melhora dos sintomas do diabetes é clara e rápida, a retirada dos medicamentos e da insulina tem que ser balanceada e lenta.

Estudos experimentais recentes ilustraram que o re-arranjo da anatomia gastrointestinal é o mediador primário do controle cirúrgico do diabetes. Os mesmos resultados foram observados em estudos investigacionais em humanos. Esses achados levantaram a possibilidade de que mecanismos do intestino proximal podem estar implicados na fisiopatologia do DMT2. Juntos, esses dados sugerem um conceito novo e revolucionário sobre uma doença antiga: DMT2 pode ser doença intestinal operável. Assim, como as novas tendências na literatura sobre diabetes, deve-se buscar a realização de estudos randomizados e controlados operação versus o melhor tratamento clínico -, focado no controle glicêmico, de lipídeos e da pressão arterial. Esses estudos, além de demonstrar o papel potencial da cirurgia para diabetes, podem definir o melhor momento para a indicação cirúrgica.

\section{REFERÊNCIAS}

1. ADA Consensus Statement - How we define cure of Diabetes? Diabetes Care, 32(11): 2133-2135, 2009

2. Ariga $\mathrm{H}$, Imai $\mathrm{K}$, Chen $\mathrm{C}$, Mantyh $\mathrm{C}$, Pappas T, Takahashi $T$ - Does ghrelin explain accelerated gastric emptying in the early stages of diabetes mellitus? Am J Physiol Regul Integr Comp Physiol 294: 
R1807- R1812, 2008

3. Broglio F, Arvat E, Benso A, Deghenghi R, Ghigo E - Ghrelin, a natural $\mathrm{GH}$ secretagogue produced by the stomach, induces hyperglycemia and reduces insulin secretion in humans. The Journal of Clinical Endocrinology \& Metabolism 86(10): 50835086, 2001

4. Buchwald $H$, Avidor $Y$, Braunwald $E$, et al. Bariatric surgery: a systematic review and meta-analysis. JAMA 2004; 292:1724 -37.

5. Cohen R, Pinheiro JS, Correa JL, Schiavon CA. Laparoscopic Rouxen-Y gastric bypass for BMI $<35 \mathrm{~kg} / \mathrm{m} 2$ : a tailored approach Surgery for Obesity and Related Diseases 2006; 2: 401-404

6. Cohen R, Schiavon CA, Pinheiro JC, Correa JL, Rubino F. Duodenaljejunal bypass for the treatment of type 2 diabetes in patients with body mass index of $22-34 \mathrm{~kg} / \mathrm{m} 2$ : a report of 2 cases. Surg Obes Relat Dis 2007; 3: 195-197.

7. Gaede $\mathrm{P}$, Lund-Andersen $\mathrm{H}$, Parving $\mathrm{HH}$, Pedersen $\mathrm{O}$ - Effect of a multifactorial intervention on mortality in Type 2 diabetes. NEJM 358:580-591, 2008

8. King H, Aubert RE, Herman WH. Global burden of diabetes, 19952025: prevalence, numerical estimates, and projections. Diabetes Care1998; 21:1414-31.

9. Laferrère B, Teixeira J, McGinty J, Tran H, Egger JR, Colarusso A, Kovack, B, Bawa B, Olivan B - Effect of weight loss by gastric bypass surgery versus hypocaloric diet on glucose and incretin levels in patients with type 2 diabetes. Journal of Clinical Endocrinology \& Metabolism, 93(7): 2479-85,2008

10. Patti ME, Houten S, Bernier R, Bianco AC, Goldfine A - Gastric bypass surgery increases bile acid levels: Potential contribution to improved glucose tolerance. Poster, American Diabetes Association Meeting, 2007, Chicago, IL

11. Pinheiro JC, Schiavon CA, Correa JL, Noujaim P, Cohen R Long-long limb Roux-en-Y gastric bypass is more efficacious in treatment of type 2 diabetes and lipid disorders in super-obese patients. Surg Obes and Relat Dis 4 (2008) 521-525, 2008

12. Pories WJ, MacDonald KG, Morgan EJ, e cols. Surgical treatment of obesity and its effect on diabetes: 10-y follow-up. Am J Clin Nutr 1992; 55 (2 Suppl): 582S-585S.

13. Rubino F, Kaplan L, Schauer P, Cummings D -The Diabetes Surgery Summit Consensus Conference Recommendations for the Evaluation and Use of Gastrointestinal Surgery to Treat Type 2 Diabetes Mellitus. Ann Surg, 2009, in press.

14. Stearns A, Balaksrishnan A, Tvakkolizadeh A- Impact of Roux-en-Y gastric bypass surgery on rat intestinal glucose transport. Am J Physiol 297:G950-G957, 2009

15. Troy $S$, Soty $M$, Ribeiro $L$, laval $L$, Migrenne $S$, Fioramonti $X$, Pillot B, Aubert R, Viollet B, Foretz M, leclerc J,Thorens B, Magnan C, Mithieux $G$, Andreeli $F$-Intestinal Gluconeogenesis Is a Key Factor for Early Metabolic Changes after Gastric Bypass but Not after Gastric Lap-Band in Mice. Cell Metabolism 8, 201-211, 2008 